\title{
Extraction of residual phospholipids from defatted soybean meal
}

\section{Paula Fernandes de Siqueira Machado ${ }^{1}$, Carlos Francisco Pedroso ${ }^{2}$, Roberto de Campos Giordano $^{3}$, Paulo Waldir Tardioli ${ }^{4}$, Raquel de Lima Camargo Giordano ${ }^{5}$}

\author{
${ }^{1,2}$ Instituto Científico e Tecnológico de Agronegócios Ltda. Rua Natal Cecone, 145, $2^{\circ}$ Andar, 81.200-330, Curitiba, PR, Brasil; \\ 1,2,3,4,5 Postgraduation Program in Chemical Engineering of the Federal University of São Carlos (PPGEQ/UFSCar), Rodovia \\ Washington Luiz, km 235, 13565-905, São Carlos, SP, Brazil; \\ 2,3,5 Chemical Engineering Department, Federal University of São Carlos, Rodovia Washington Luiz, km 235, PO Box 676, 13565-905, \\ São Carlos, SP, Brazil.
}

Email: icta.pesquisa@gmail.com, carlos@intecso.com.br, raquel@ufscar.br,pwtardioli@ufscar.br

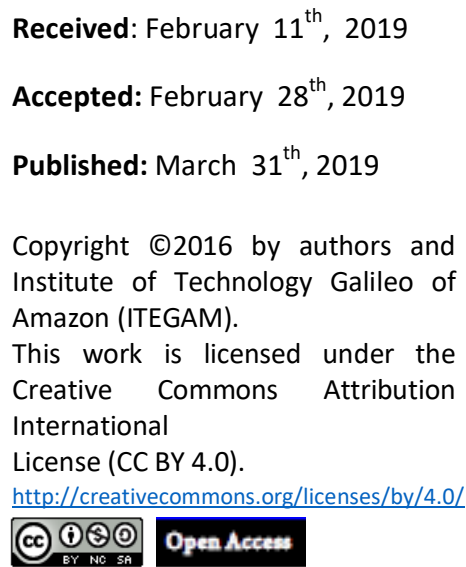

Received: February $11^{\text {th }}, 2019$

Accepted: February $28^{\text {th }}, 2019$

Published: March $31^{\text {th }}, 2019$

Copyright (C2016 by authors and Institute of Technology Galileo of Amazon (ITEGAM).

This work is licensed under the Creative Commons Attribution International

License (CC BY 4.0).

http://creativecommons.org/licenses/by/4.0/ (c) (i) (2) (2) Open Aeces:

\section{ABSTRACT}

Phospholipids are the main components of commercial lecithin, which is widely used in food and pharmaceutical industries. This work studies the extraction of residual phospholipids (PLs) from soybean meal, which is produced after soy oil extraction with hexane. The extraction efficiency was evaluated with four systems: hexane, hexane:ethanol (4:1, v/v), chloroform:methanol (2:1, v/v), and ethanol:water (3:1, v/v). Ethanol:water and hexane:ethanol were more effective, presenting similar extraction yield. The ethanol:water relation was then varied from 30 to $90 \%(\mathrm{v} / \mathrm{v})$, and the best extraction conditions were 70 and $90 \%(\mathrm{v} / \mathrm{v})$, with maximum yield of $1.50 \mathrm{~g} / 100 \mathrm{~g}$. Although, the highest PLs solubility, $6.225 \mathrm{~g} / \mathrm{L}$, was observed for $90 \%$ ethanol, at $75^{\circ} \mathrm{C}$. This work allowed confirming the presence of a significant amount of residual phospholipids in soybean meal, which can be recovered using different solvent systems and processed in products with high commercial value.

Keywords: soybean meals, residual phospholipids, lecitina, solid-liquid extraction.

\section{Extração de fosfolipídio residual a partir de farelo de soja desengordurado}

\section{RESUMO}

Fosfolipídios são os principais componentes da lecitina comercial, substância amplamente utilizada na indústria de alimentos, devido as suas propriedades emulsificantes. Neste trabalho foram avaliados os rendimentos da extração de fosfolipídios residuais a partir do farelo de soja, produto gerado após a extração hexânica do óleo do grão de soja. A eficiência de extração foi avaliada em quatro sistemas: hexano; hexano:etanol (4:1, v/v), clorofórmio:metanol (2:1, v/v) e etanol: água (3:1, $\mathrm{v} / \mathrm{v})$. As misturas etanol:água e hexano:etanol foram mais eficientes, apresentando taxas similares de extração. A proporção ideal etanol: água também foi avaliada e as melhores extrações foram obtidas com $70 \%$ e $90 \%$ de etanol (v/v), chegando a taxas máximas de $1,5 \mathrm{~g} / 100 \mathrm{~g}$, enquanto que a melhor solubilidade do fosfolipídio foi em $90 \%, 6,225 \mathrm{~g} / \mathrm{L}$ a $75^{\circ} \mathrm{C}$. Esses resultados confirmam a presença significativa de fosfolipídios residuais, os quais podem ser facilmente recuperados e processado para obtenção de produtos com alto valor comercial

Palavras-chave: farelo de soja, fosfolipídio residual, lecitina, extração sólido-líquido.

\section{INTRODUTION}

Fosfolipídios (PL) são lipídios polares [1] de grande interesse das indústrias de alimentos e farmacêutica devido ao poder emulsificante e a sua importância nutricional [2]. São os principais constituintes das lecitinas comerciais. Apesar da terminologia não ser a mais exata, tornou-se habitual nos círculos industriais chamar de lecitina a mistura de fosfolipídios, triglicerídeos, lipídios complexos e carboidratos obtida industrialmente pelo processo de degomagem de óleos vegetais 
brutos [3]. As Lecitinas podem ser obtidas da gema de ovo e de várias sementes oleaginosas, tais como linho, algodão, germe de milho, girassol, colza, e soja [4]. O grão de soja é a fonte vegetal predominante de lecitina, devido a abundância de fosfolipídios em sua constituição [5].

Sackeheim e Lehman [6] dividem os fosfolipídios em duas categorias: fosfoglicerídeos (ou glicerofosfolipídios, [7]) e fosfoesfingosídeos (ou esfingomielinas, [7]), dependendo de sua porção polar. Nos fosfoglicerídeos, o glicerol forma um éster com dois ácidos graxos adjacentes e uma unidade fosfato ligada a um substituinte derivado de um álcool de baixo peso molecular [8].

Como os fosfoglicerídeos apresentam duas cadeias hidrofóbicas longas e um grupo polar (hidrofílico), são moléculas anfipáticas capazes de formar micelas em água [6, 8]. Os fosfolipídios mais abundantes nas lecitinas são: fosfatidilcolina (PC), fosfatidiletanolamina (PE) ácido fosfatídico (PA) e fosfatidilinositol (PI) [9]. O grupo de Beare-Rogers [10] em trabalho colaborativo interlaboratorial publicado junto à IUPAC determinaram a composição de concentrados de lecitina de soja comercialmente disponíveis, em relação aos níveis de fosfatidiletanolamina $(9,45-15,80 \%)$, ácido fosfatídico $(4,68-$ $12,58 \%)$, fosfatidilinositol $(7,67-12,43 \%)$ e fosfatidilcolina $(9,33-19,01 \%)$, por cromatografia líquida de alta eficiência.

Atualmente, o solvente utilizado para extração de óleo de sementes oleaginosas é o hexano [11]. O óleo bruto, obtido por extração direta por hexano, contem aproximadamente $2-3 \%$ de fosfolipídios. Este óleo é degomado mediante hidratação dos fosfolipídios com água e separação dos mesmos por centrifugação. As gomas separadas podem ser incorporadas aos farelos proteicos de soja ou secas dando origem às lecitinas comerciais tradicionais [12]. Diversos estudos tem demonstrado a baixa eficiência do hexano na remoção de lipídios polares [13-17].

Segundo Hayes e colaboradores [16], a quantidade de lipídios residuais no farelo oscila entre 0,5 a $5 \%$ do seu peso seco. Para a extração de lipídios residuais do farelo, os autores sugerem a utilização de um meio de extração contendo um solvente hidrocarboneto (hexano, n-heptano, naftas, parafinas, e misturas) e um álcool monoídrico (metanol, etanol, isopropanol, n-propanol, butanol, isobutanol, n-butanol e misturas desses). Se a concentração do álcool for muito baixa $(<10 \%)$ a eficiência de remoção é reduzida, se for muito alta $(>70 \%)$, haverá uma significativa remoção de outros componentes, como açúcares. É desejável que a umidade da mistura esteja entre 5 e $15 \%$, não ultrapassando esse valor.

Os lipídios tem grande faixa de hidrofobicidade sendo praticamente inviável a utilização de um único solvente universal. Lipídios neutros contêm apenas ligações covalentes, sendo facilmente extraídos por solventes apolares, enquanto os lipídios polares estão unidos por forças eletrostáticas e pontes de hidrogênio necessitando, portanto, de um solvente polar capaz de competir com tais interações [17]. A mistura de solvente ideal para extração de matéria graxa de tecidos deve ser suficientemente polar para removê-los das associações com as membranas celulares ou com lipoproteínas, sem que ocorra reação química. Os métodos que utilizam a mistura binária clorofórmio e metanol para extração de lipídios têm como vantagem a capacidade de extraírem tanto os lipídios neutros quanto os lipídios polares eficientemente, no entanto, a toxicidade destes solventes limita a utilização para escala de bancada apenas.
O presente estudo foi realizado com intuito de avaliar diferentes sistemas de solventes para a extração de fosfolipídios em farelo de soja e comparar os sistemas testados ao solvente comumente utilizado em extração de óleo, o hexano. O comportamento destes compostos foi estudado em diferentes concentrações de etanol e a condição de saturação dos principais fosfolipídios foi determinada para cada concentração etanólica em diferentes temperaturas.

\section{DESENVOLVIMENTO}

Para os experimentos de extração de fosfolipídios utilizouse farelo de soja desengordurado e tostado Imcosoy 48, produzido pela Imcopa - Importação, Exportação e Indústria de Óleos S.A. Os testes de solubilidade da lecitina em pó foram realizados com Lecitina em Pó Solec F, produzida pela Solae do Brasil - Indústria e Comércio de Alimentos Ltda. Os solventes utilizados foram todos de grau analítico.

\section{II.1 MÉTODOS ANALÍTICOS}

As análises de proteína bruta, umidade e lipídios foram realizadas no laboratório de controle de qualidade da Imcopa Importação, Exportação e Indústria de Óleos S.A. A análise de proteína bruta foi realizada pelo método Kjeldahl, conforme descrito na monografia $\mathrm{Bc}$ 4-91 da AMERICAN OIL CHEMIST'S SOCIETY [18]. A análise de umidade foi realizada pela perda de massa do farelo a temperatura de $130^{\circ} \mathrm{C}$ por três horas, conforme descrito na monografia Ac 2-41 da AMERICAN OIL CHEMIST'S SOCIETY [18]. A análise de lipídios foi realizada por extração por solvente orgânico sob aquecimento por 3 horas conforme descrito na monografia $\mathrm{Ba}$ 3-38 da AMERICAN OIL CHEMIST'S SOCIETY [18].

As análises de açúcares e fosfolipideos foram realizadas em cromatografo líquido de alta eficiência (CLAE) Agilent 1200 series e Software Chemstation.

Os açúcares foram analisados com detecção por índice de refração. A coluna usada foi Supelcogel Ca 300 x 7,8 mm com pré-coluna Supelcoguard CA 50 x 4,6 mm da Supelco. A fase móvel foi água ultra pura. As condições cromatográficas empregadas foram: volume de injeção de $5 \mu \mathrm{L}$, fluxo da fase móvel $0.3 \mathrm{~mL} / \mathrm{min}$, temperatura do forno de coluna $80^{\circ} \mathrm{C}$, tempo de corrida de 35 minutos

Os teores de fosfolipídios foram quantificados por cromatografia liquida em fase normal com detecção de espalhamento de luz evaporativo. Foi usada uma coluna de sílica modelo Varian Pursuit XRS Si 100 x 4,6 mm, 3.0 um, com uma temperatura de forno de $40{ }^{\circ} \mathrm{C}$. A fase móvel usada foi uma mistura de solventes orgânicos divididos em dois grupos. A fase móvel A: n- hexano, 2-propanol, ácido acético glacial, trietilamina $(820 / 165 / 10 / 0,8 \mathrm{v} / \mathrm{v} / \mathrm{v} / \mathrm{v})$ e a fase B composta por $2-$ propanol, água ultrapurificada, ácido acético glacial, trietilamina $(845 / 140 / 1,0 / 0,8 \mathrm{v} / \mathrm{v} / \mathrm{v} / \mathrm{v})$

\section{II.2 EXPERIMENTOS LABORATORIAIS}

Testes em escala de laboratório foram planejados para se estudar a extração de fosfolipídios remanescentes no farelo de soja após extração do óleo bruto da soja com hexano. O primeiro experimento foi conduzido para avaliar a eficiência de extração de quatro diferentes sistemas de solventes: hexano, hexano:etanol, 
etanol aquoso e clorofórmio:metanol, estabelecendo-se o sistema de solvente mais adequado para se obter o maior rendimento de extração. Com o intuito de verificar se a concentração do sistema de solventes selecionada no primeiro teste era a mais adequada ( $75 \% \mathrm{v} / \mathrm{v}$ etanol aquoso), um segundo experimento foi conduzido variando-se a concentração de etanol utilizada em solução aquosa. Ainda utilizando como solvente a solução alcoólica, o terceiro experimento foi realizado com objetivo de avaliar as solubilidades dos fosfolipídios de acordo com a concentração alcoólica e a temperatura, sendo que neste experimento a lecitina em pó foi utilizada como matéria prima por apresentar um teor elevado de fosfolipídios.

Uma amostra de farelo desengordurado de soja, aproximadamente $2 \mathrm{~kg}$, foi coletada e moída em moinho analítico padronizando sua granulometria abaixo de 0,50mm (35 mesh). Analisaram-se os teores de umidade, proteína bruta, lipídios e açúcares.

Uma alíquota de aproximadamente $100 \mathrm{~g}$ foi colocada em papel filtro qualitativo procurando-se minimizar a compactação. $\mathrm{Na}$ parte superior do papel foi colocada uma porção de algodão para promover melhor distribuição do solvente no leito. A amostra foi então colocada em extrator de Soxhlet de $500 \mathrm{~mL}$.

Em um balão de fundo arredondado foram colocados os solventes de extração para cada experimento: etanol $75 \%$, hexano:etanol (4:1), hexano e clorofórmio:metanol (2:1). O experimento foi realizado em capela com exaustão.

As amostras foram extraídas exaustivamente por 24 horas, procurando garantir assim uma extração completa dos fosfolipídios possíveis pelo solvente. Após a extração o farelo o solvente foi removido em estufa com circulação de ar a $75^{\circ} \mathrm{C}$, enquanto o material extraído foi concentrado em evaporador rotativo Fisatom modelo 803 a aproximadamente $60^{\circ} \mathrm{C}$ e 500 $\mathrm{mmHg}$. Os procedimentos de extração foram realizados em triplicata.

No farelo desengordurado extraído procederam-se análises de proteína, umidade e lipídios. No farelo extraído com etanol $75 \%$ analisou-se também o teor de açúcares. Nos extratos concentrados quantificou-se o teor de fosfolipídios.

A extração foi realizada em um balão de extração aquecido e agitado por chapa aquecedora magnética e conectado a um condensador. As condições da extração foram: temperatura de ebulição do solvente; farelo desengordurado moído, com granulometria inferior a $0,5 \mathrm{~mm}$; massa inicial de farelo igual a $100 \mathrm{~g}$; volume de solvente de 1,6 litros; tempo de extração de 45 minutos, considerados a partir do início da ebulição do solvente; agitação constante.

Após a extração filtrou-se o farelo em papel filtro qualitativo, com auxílio de bomba de vácuo e funil de Büchner, e em seguida lavou-se o farelo com $300 \mathrm{~mL}$ de solvente limpo, na temperatura de ebulição. $\mathrm{O}$ farelo foi acondicionado em copo de Becker tarado e seco em estufa de circulação para posterior pesagem. O solvente, contendo os extratos, foi filtrado para remoção de qualquer resíduo sólido, concentrado em evaporador rotativo e pesado para análise dos teores de proteína e umidade dos farelos residuais e o teor de fosfolipídios.

Primeiramente, estudou-se o comportamento da lecitina em pó quando solubilizada em diferentes sistemas solventes variando-se a concentração alcoólica de zero (água deionizada) a
$100 \%$ (etanol anidro). O estudo foi realizado em temperatura ambiente, pois se entende que o aumento de temperatura aumentará a solubilidade em qualquer proporção de solvente testada.

Adicionou-se aproximadamente $1 \mathrm{~g}$ de lecitina em pó e 100 $\mathrm{mL}$ de solução alcoólica, anotando-se os pesos exatos da lecitina pesada e da mistura final em balança analítica. A solubilização foi feita em Erlenmeyer de $250 \mathrm{~mL}$, por duas horas em mesa agitadora orbital, mantida a $25^{\circ} \mathrm{C}$. Após solubilização as amostras foram filtradas em papel filtro qualitativo e em seguida centrifugadas a $4000 \mathrm{rpm}$ por 10 minutos, pois se observou a presença de precipitados em algumas das amostras após a filtração. Os sobrenadantes foram enviados para análise de teor de fosfolipídios.

Obtido o perfil de solubilidade da lecitina em relação ao grau alcoólico, escolheram-se os pontos de maior e menor solubilidade e um ponto intermediário para estudo dos perfis de solubilidade em função da temperatura $\left(25-75^{\circ} \mathrm{C}\right)$. Em balão de fundo chato colocou-se $17 \mathrm{~g}$ de lecitina em pó e 1,7 litros de solvente. As concentrações de etanol testadas foram 50, 75 e $90 \%$. Ao balão de fundo chato acoplou-se um condensador e todo o sistema foi levado ao aquecimento em agitador magnético por duas horas. Cada solvente foi testado nas temperaturas de 25, 40, 50,60 e $75^{\circ} \mathrm{C}$. Posteriormente, as amostras foram transferidas para tubos de centrífuga, sendo submetidas à centrifugação a $4.000 \mathrm{rpm}$ por cerca de 10 minutos na temperatura de extração. Os sobrenadantes das amostras foram filtrados com auxílio de filtros qualitativos. As análises foram realizadas em triplicatas para cada variável testada.

\section{RESULTADOS E DISCUSSÃO}

\section{III.1 INFLUÊNCIA DE DIFERENTES SOLVENTES NAS EFICIÊNCIAS E SELETIVIDADE DA EXTRAÇÃO DE FOSFOLIPÍDIOS RESIDUAIS NO FARELO DESENGORDURADO}

Os resultados obtidos em 24 horas de extração etanol $75 \%$, hexano, etanol: hexano (4:1) e clorofórmio: metanol (2:1) em Soxhlet estão mostrados na Tabela 1. Observa-se que os solventes com maiores capacidades de extração dos fosfolipídios do farelo de soja desengordurado foram o etanol $75 \%$ e a mistura hexano: etanol. Os rendimentos obtidos (expressos em massa de fosfolipídios por massa de farelo desengordurado seco) foram $1,48 \%$ para o etanol $75 \%$ e $1,44 \%$ para a mistura hexano:etanol, enquanto para o hexano, solvente usualmente empregado pelas empresas esmagadoras de soja, foi de $0,43 \%$. Os resultados de rendimento mássico da extração indicam que tanto etanol $75 \%$ quanto a mistura hexano:etanol parecem ser igualmente satisfatórios para a extração do teor residual de fosfolipídios. Contudo, se analisarmos a composição dos extratos obtidos, verifica-se que com etanol $75 \%$ obteve-se $8,10 \%$ de fosfolipídios em relação à massa de extrato seco, enquanto o extrato obtido com a mistura hexano: etanol teve 40,61\% (massa de fosfolipídios/massa de extrato seco). Essa diferença decorre, devido a extração conjunta de fosfolipídios e açúcares pelo solvente etanol $75 \%$. Portanto, a mistura hexano:etanol permitiu atingir maior grau de pureza do extrato. 
Tabela 1: Rendimentos de extração de fosfolipídios obtidos para diferentes solventes com 24 horas de extração em Soxhlet.

\begin{tabular}{|ccc|}
\hline Solvente & $\begin{array}{c}\text { Rendimento } \\
\text { de extração } \\
\text { \% }\end{array}$ & $\begin{array}{c}\text { Teor de PL no } \\
\text { extrato seco }\end{array}$ \\
\hline Hexano & $0,43 \pm 0,01$ & $34,81 \pm 1,45$ \\
Hexano:Etanol (4:1) & $1,44 \pm 0,03$ & $40,61 \pm 4,64$ \\
Clorofórmio:Metanol & $1,08 \pm 0,07$ & $22,05 \pm 1,89$ \\
$(2: 1)$ & $1,48 \pm 0,01$ & $8,11 \pm 0,11$ \\
\hline Etanol $75^{\circ} \mathrm{GL}$ & & \\
\hline
\end{tabular}

Fonte: Autores, (2019).

Os sistemas clorofórmio: metanol e hexano:etanol foram escolhidos com base nos trabalhos encontrados na literatura [15-17]. O etanol em solução aquosa foi escolhido devido à sua utilização na produção de concentrados proteicos [19]. Adicionalmente, a utilização do etanol como solvente para extração de lipídios tem recebido atenção por ser menos tóxico e mais sustentável em relação aos solventes derivados de petróleo [20-21]. A qualidade da lecitina também está relacionada ao perfil de fosfolipídios, sendo importante a presença de fosfolipídios de diferentes polaridades e por esse motivo foi realizada a otimização o processo de extração de fosfolipídios em etanol aquoso

A Figura 1 mostra uma comparação entre os rendimentos de extração de cada solvente para os quatro principais fosfolipídios (PC, PI, PE e PA) e para o somatório dos PLs minoritários. Para todos os solventes, a fosfatidilcolina (PC) é o fosfolipídio majoritário, seguida do fosfatidilinositol (PI). As relações entre os teores de fosfolipídios de cada extrato foram muito similares, com exceção do etanol $75 \%$, no qual se obteve maior extração de PI e menor extração de PA e dos PLs minoritários em relação aos fosfolipídios totais. O clorofórmio:metanol apresentou acentuada seletividade para os PLs minoritários. Conforme relatado por Brum et al. [17], Nielsen [15] e Hayes et al. [16] todas as misturas que contendo solventes polares foram superiores ao hexano na extração dos fosfolipídios. Segundo Hayes et al. [16] o álcool monoídrico não funciona somente como um co-solvente, mas como um agente que reduz a tensão superficial do solvente, aumentando sua capacidade de penetração e promovendo a liberação de lipídeos do complexo proteína-carboidrato. Em desacordo com o relatado por Brum et al. [17], a mistura clorofórmio:metanol foi menos eficiente na extração dos fosfolipídios que mistura hexano:etanol. Também contrariando o relatado por Hayes et al. [16] a extração com etanol aquoso foi tão eficiente quanto o hexano:etanol, podendo ser utilizada como única etapa de extração para obtenção de lecitina e concentrado proteico.

Comparando-se o perfil de PLs dos extratos etanol $75 \%$ e hexano:etanol (4:1), que tiveram eficiências de extração similares, observa-se que o etanol $75 \%$ extraiu seletivamente maiores quantidades de PI e menores quantidades de PA e PLs minoritários (Figura 1). Em contrapartida, o hexano:etanol teve menor eficiência de extração para o PI e maior para o PA e os PLs minoritários. Considerando que os maiores rendimentos de extração foram obtidos para as misturas etanol:água e hexano:etanol e que os rendimentos de extração não foram os mesmos para alguns fosfolipídios, pode-se afirmar que nenhum dos solventes foi capaz de extrair 100\% dos PLs existentes no farelo se soja desengordurado.

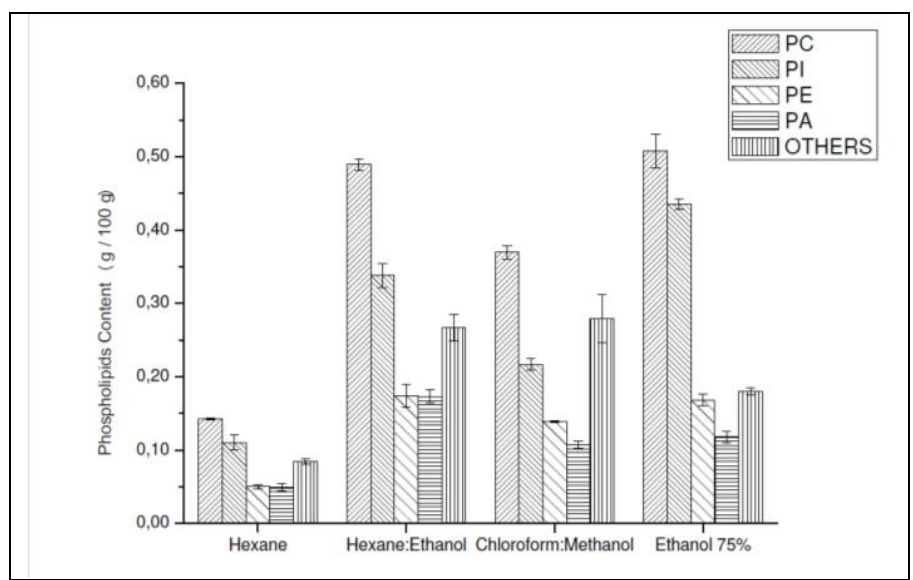

Figura 1: Perfil de fosfolipídios (PL) obtido para cada solvente testado.

Fonte: Autores, (2019).

A partir dos perfis de fosfolipídios obtidos observou-se que a mistura etanol: água extraiu em maior grau fosfolipídios mais polares, como PI, e em menor grau os menos polares, como o PA. A polaridade do fosfolipídio está diretamente associada com o seu poder emulsificante, sendo o PC, o PI e o PE os fosfolipídios de maior importância. A presença de altas concentrações de PA e dos PLs minoritários reduz a qualidade da lecitina. Com o intuito de verificar se a concentração da solução alcoólica selecionada neste estudo foi a mais adequada $(75 \% \mathrm{v} / \mathrm{v})$, um segundo experimento foi conduzido variando-se a concentração de etanol em solução aquosa entre 30-90\% (v/v).

\section{III.2 INFLUÊNCIA DA PROPORÇÃO ETANOL:ÁGUA NA EFICIÊNCIA E SELETIVIDADE DA EXTRAÇÃO DE FOSFOLIPÍDIOS RESIDUAIS DO FARELO DESENGORDURADO}

Como podemos observar na Figura 2, a extração dos fosfolipídios totais do farelo de soja desengordurado foi aumentando conforme o aumento da proporção de etanol no solvente, com os melhores resultados obtidos entre 70 e $80 \%$. Pode ser observado que o rendimento aumentou com o aumento da concentração de etanol no sistema, atingindo um rendimento máximo de $1,53 \mathrm{~g} / 100 \mathrm{~g}$ na condição de $70 \%$ de etanol.

A Figura 3 mostra os rendimentos das extrações dos fosfolipídios PC, PI, PE, PA e o somatório dos demais PLs com a variação da concentração de etanol. Podemos verificar, os fosfolipídios isoladamente, que os fosfolipídios PC, PE, PA e somatório dos demais PLs apresentaram o mesmo comportamento descrito quando olhamos a soma total dos fosfolipídios (Figura 2), ou seja, atingiram o ponto máximo de extração quando o solvente apresentou uma concentração de 70\%. Apenas o PI mostrou uma queda significativa quando a concentração alcoólica aumentou de 80 para $90 \%$, o que está de acordo com a literatura disponível. De acordo com Wu and Wang [22], o PI apresenta menor solubilidade em etanol que os demais fosfolipídios. 


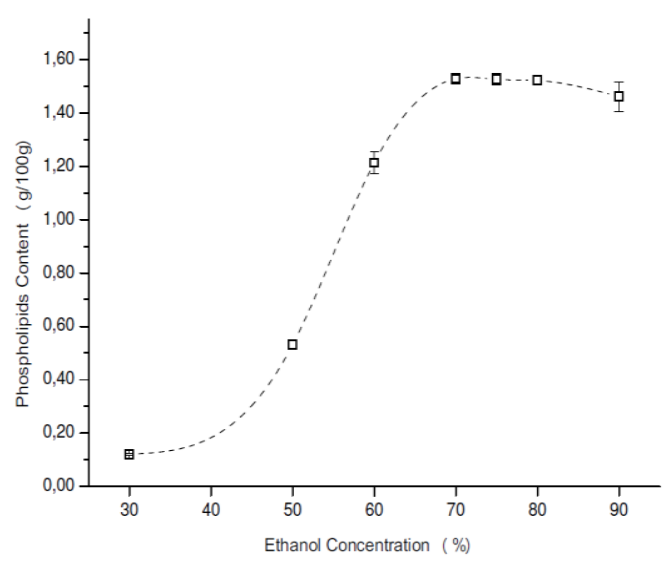

Figura 2: Rendimentos de extração de fosfolipídios do farelo desengordurado de soja em função do teor de etanol no solvente de extração.

Fonte: Autores, (2019).

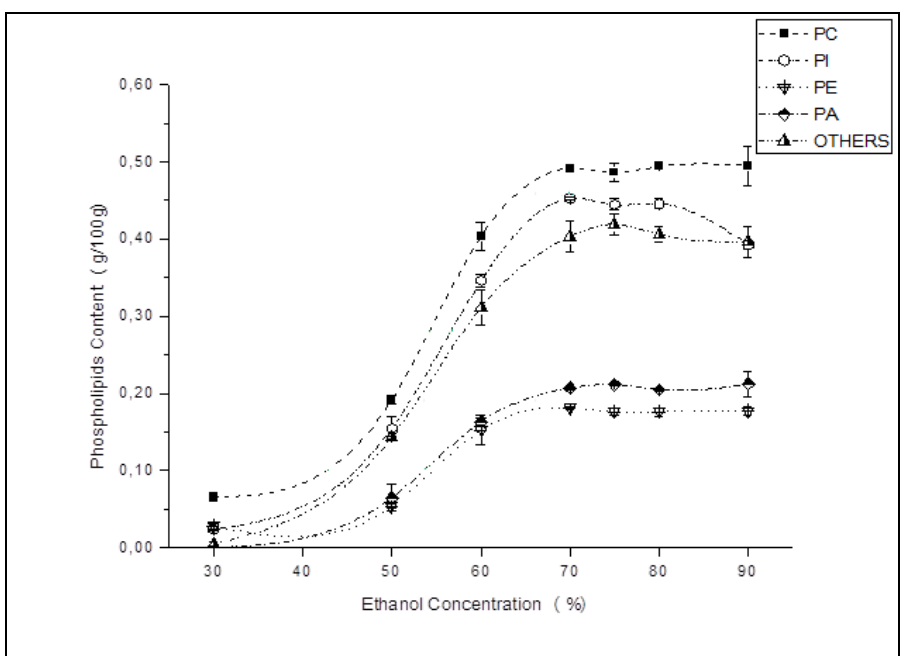

Figura 3: Variação dos rendimentos nas extrações dos principais fosfolipídios em função do teor alcoólico. Resultados expressos em fosfolipídios por farelo desengordurado seco $(\mathrm{g} / 100 \mathrm{~g})$.

Fonte: Autores, (2019).

\section{III.3 ESTUDO DA SOLUBILIDADE DE LECITINA EM PÓ COMERCIAL EM DIFERENTES PROPORÇÕES DE ETANOL:ÁGUA EM DIFERENTES TEMPERATURAS}

Segundo Kaparthi e Chari [23], uma abordagem racional para o projeto de um extrator eficiente para extrair óleos vegetais de materiais oleaginosos requer um conhecimento das solubilidades do óleo no solvente proposto. Os dados publicados sobre as solubilidades dos óleos em etanol são escassos. Não foram encontrados trabalhos na literatura a respeito da solubilidade das lecitinas.

Por natureza lipídica, fosfolipídios não são solúveis em água, mas formam emulsões estáveis. A ocorrência deste fenômeno pode ser observada através da turbidez obtida nos ensaios com teor alcoólico inferior a $20 \%(\mathrm{v} / \mathrm{v})$. A partir de $30 \%$ de etanol observou-se a presença de uma pequena quantidade de precipitado sólido durante a centrifugação, mas o sobrenadante ainda se mostrava turvo. A partir de $50 \%$ pode-se verificar a presença de grande quantidade de material precipitado, com o sobrenadante se mostrando límpido, podendo-se afirmar que o material quantificado na fase líquida era realmente lecitina dissolvida. Nesta condição apenas $0,41 \mathrm{~g} / \mathrm{L}$ de fosfolipídios, dos
7,03 g/L adicionados, foram encontrados em solução. A partir desse ponto, a solubilidade aumentou linearmente com a concentração de etanol na solução até atingir a máxima solubilidade em $90 \%$ de etanol - 4,93 g/L. Em solução de etanol anidro a solubilidade sofreu um decréscimo para $4,14 \mathrm{~g} / \mathrm{L}$.

Outra observação importante foi que a $50 \%$ de etanol pode-se precipitar $94,28 \%(\mathrm{~m} / \mathrm{m})$ dos fosfolipídios presentes. Esse fenômeno pode ser utilizado como estratégia para futura recuperação da lecitina extraída

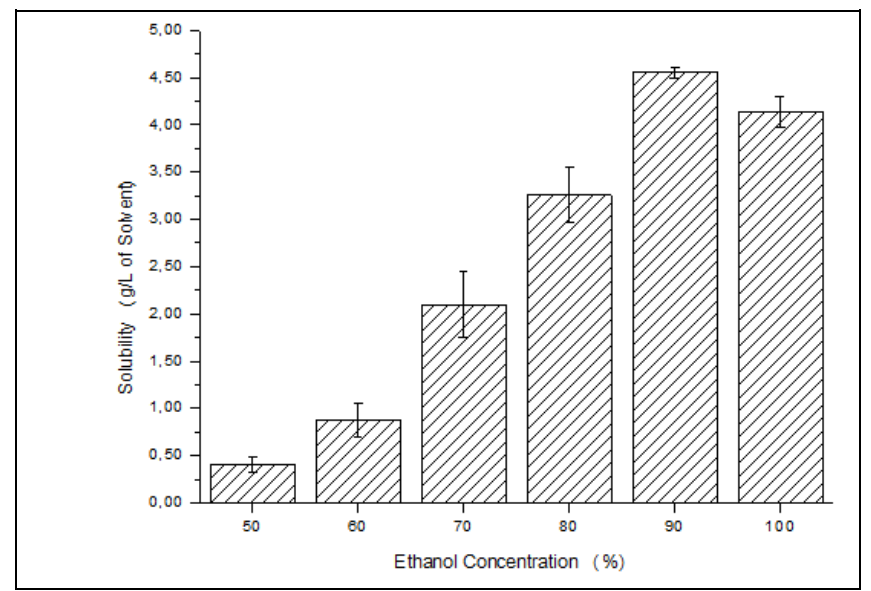

Figura 4: Solubilidade dos fosfolipídios totais presentes na lecitina em pó em função da concentração alcoólica. Resultados expressos em gramas de fosfolipídios totais por litro de solvente.

Fonte: Autores, (2019).

A Figura 5 mostra o perfil de solubilidade dos fosfolipídios individualmente. Nota-se que todos se comportam de maneira semelhante, sendo a fosfatidilcolina (PC) a mais influenciada pelas alterações do teor alcoólico. Todos os fosfolipídios aumentaram a solubilidade com o aumento da concentração alcoólica, atingindo a solubilidade máxima em $90 \%$.

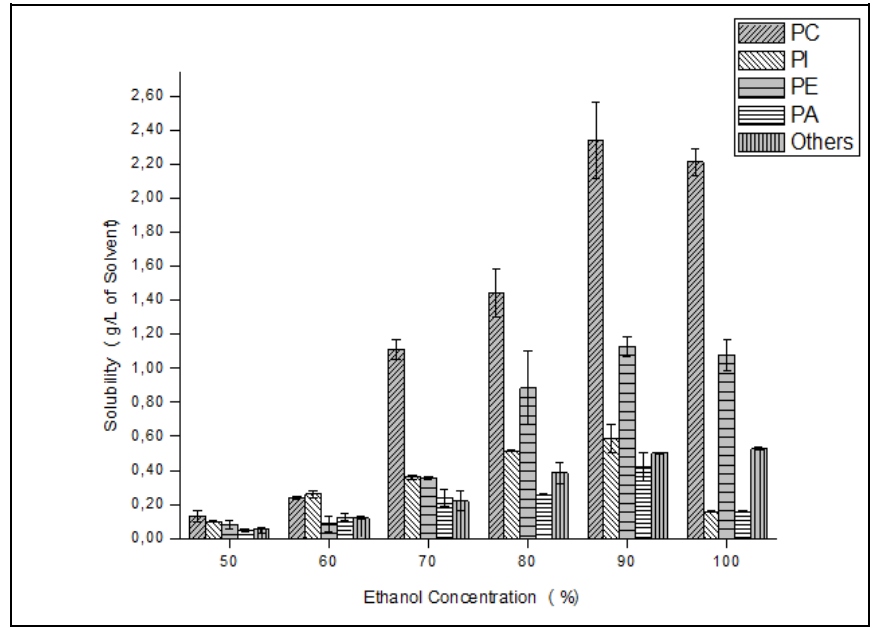

Figura 5: Perfil de solubilidade dos principais fosfolipídios em função do teor alcoólico.

Fonte: Autores, (2019).

Em etanol anidro $(99,3 \%)$ apenas as solubilidades do PI e do PA mostraram reduções significativas. De acordo com Wu e Wang (2003), PC é muito solúvel em etanol, enquanto que a solubilidade de PI é significativamente menor em solução etanólica. O PC em etanol $90 \%$ e anidro foi totalmente solubilizado, ou seja, as 2,22 $\mathrm{g} / \mathrm{L}$ adicionadas foram dissolvidas. 
A seguir, investigou-se a influência da temperatura na solubilidade dos fosfolipídios. Selecionaram-se as concentrações de 50, 75 e $90 \%$ de etanol para avaliação do perfil de solubilidade a diferentes temperaturas.

A Figura 6 indica que, conforme esperado, a solubilidade dos fosfolipídios aumenta com o aumento da temperatura para as três concentrações de etanol testadas. A solubilidade mínima observada foi de $0,499 \mathrm{~g} / \mathrm{L}$, em $50 \%$ de etanol a $25^{\circ} \mathrm{C}$, e máxima de $6,225 \mathrm{~g} / \mathrm{L}$ em $90 \%$ de etanol a $75^{\circ} \mathrm{C}$. As concentrações de saturação do PC não foram determinadas devido a total solubilidade deste em etanol $90 \%$.

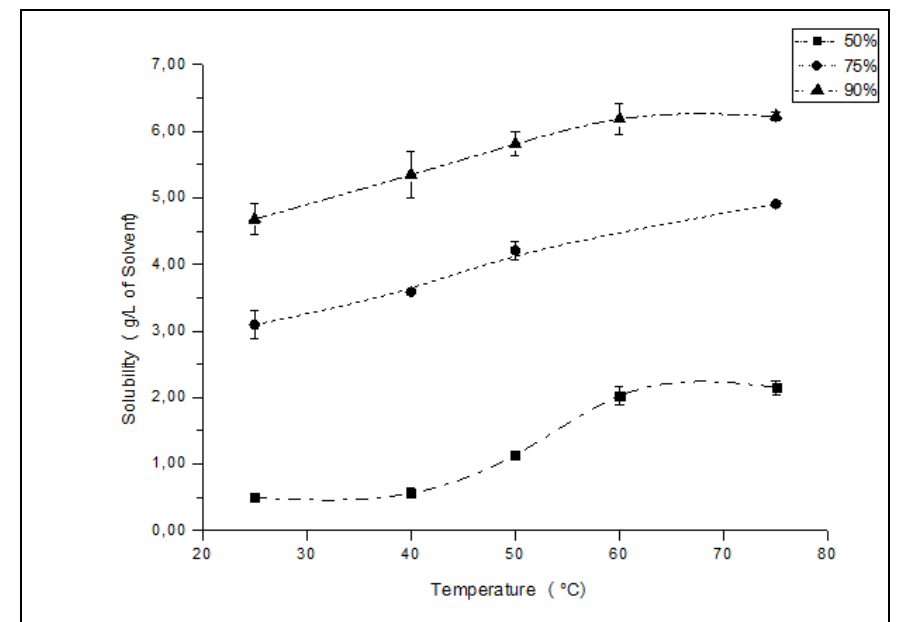

Figura 6: Solubilidades dos fosfolipídios totais em função da temperatura nas concentrações alcoólicas fixas de 50, 75 e $90 \%$.

Fonte: Autores, (2019).

\section{CONCLUSÕES}

Os experimentos de extração realizados neste trabalho comprovaram a existência de expressivo teor residual de fosfolipídios no farelo de soja desengordurado. Testes utilizando diferentes solventes mostraram maiores eficiências de extração com etanol $75 \%$ e com a mistura hexano:etanol, sendo que a segunda apresentou uma maior pureza do extrato. Em contrapartida, na extração com etanol $75 \%$ obteve-se um perfil de fosfolipídios mais conveniente devido à presença em maior quantidade de fosfolipídios de maior polaridade, como fosfatidilcolini, fosfatidiletanolamina e fosfatidilinsitol. $\mathrm{O}$ estudo da influência da proporção etanol:água mostrou que o rendimento da extração de fosfolipídios aumenta, com o aumento da concentração de etanol no sistema. Os resultados mostraram que na faixa de 50 a $90 \%$ de etanol a solubilidade aumenta. A influência da temperatura na faixa de 25 a $75^{\circ} \mathrm{C}$ foi concomitantemente avaliada, apresentando uma relação direta entre temperatura e solubilidade. Das condições testadas, o menor valor de solubilidade encontrado foi de $0,499 \mathrm{~g} / \mathrm{L}$ em etanol $50 \%$ a $25^{\circ} \mathrm{C}$. A maior solubilidade foi observada foi de $6,225 \mathrm{~g} / \mathrm{L} \mathrm{em}$ $90 \%$ de etanol e $75^{\circ} \mathrm{C}$. Vale lembrar que a extração não depende apenas da solubilidade, mas também com a penetração do solvente e seu poder emulsionante.

$\mathrm{O}$ processo de recuperação da lecitina utilizando a precipitação com etanol será estudado em trabalhos futuros utilizando o farelo desengordurado como matéria-prima.

Após a conclusão dos experimentos confirmamos a existência de uma expressiva quantidade de fosfolipídios residuais no farelo de soja, mesmo após a etapa de extração da soja com hexano. Estes fosfolipídios podem ser recuperados através de ensaios rápidos e de baixo custo e transformados em lecitina de soja, produto de alto valor agregado para os produtos derivados de soja.

\section{AGRADECIMENTOS}

Os autores agradecem ao Instituto Científico e Tecnológico em Agronegócio, a Imcopa - Importação, Exportação e Indústria de Óleos S.A., Intecso Soluções e Inovações em Agronegócio pelos serviços prestados e ao Conselho Nacional de Pesquisa, CNPq pelo apoio a essa pesquisa e sua continuidade.

\section{REFERÊNCIAS}

[1] L.A. Horrocks, Nomenclature and structure of phospholipides, in: Szuhaj, B.F., Lecithins: Sources, Manufacture \& Uses, AOCS PRESS: Champaign, p. 1-6, 1989.

[2] C.R. Scholfield, Occurrence, structure, composition, in: Szuhaj, B.F. List, G.R., Lecithins, AOCS PRESS: Champaign, p. 1-20, 1985 .

[3] V. Wywiol; M. Glatz; F. Müller, Impuls: Information for customers, partners and employees. Hamburg: Stern Lecithin \& Soja GmbH \& Co. KG. Abr., 1994. 5 p.

[4] W.V. Nieuwenhuyzen, Lecithin production and properties. Journal of American Oil Chemists' Society, Hamburg, v. 53, p. 425-427, jun. 1976.

[5] J.P. Cherry W.H. Kramer,Plant Sources of Lecithin, in: Szuhaj, B.F., Lecithins: Sources, Manufacture, \& Uses, AOCS PRESS: Champaign, p. 16-31, 1989.

[6] G.I. Sackheim; D.D. Lehman, Química e bioquímica para ciências biomédicas. 8.ed. São Paulo: Manole, 2001.

[7] A.J. Leonel, Produção e caracterização de partículas lipídicas constituídas por ácidos graxos, gordura vegetal hidrogenada e lecitina na encapsulação de um composto hidrofílico de baixa massa molar. Faculdade de Engenharia de Alimentos, da Universidade Estadual de Campinas, 2008.

[8] K.P. Vollhardt; N. E. Schore, Química orgânica. Estrutura e função. $4^{\mathrm{a}}$ ed. Porto Alegre: Bookman, 2004.

[9] W.V. Nieuwenhuyzen; M.C. Tomás Update on vegetable lecithin and phospholipid technologies. European Journal of Lipid Science and Technology, Limmen, v. 110, p. 472-486, 2008.

[10], J.L. Beare-Rogers, A. Bonekamp-Nasner; A. Dieffenbacher Determination of the phospholipid profile of lecithins by high performance liquid chromatography. Technical Report IUPAC, Pure \& Appl. Chern., Vol. 64, No. 3, pp. 447-454, 1992.

[11] L.A. Johnson, E. W Lusas. Comparison of alternative solvents for oils extraction. J. Am. Oil Chem. Soc., v. 60, n. 2, p. 229-242, 1983. 
[12] H. Rittner Sementes oleaginosas: subprodutos e resíduos de extração. Tecnologia matérias graxas: Extração, v. 1, p. 9811074, 2002..

[13] L. Montanari, P. Fantozzi, J.M. Snyder, J.W. E King, Selective extraction of phospholipids from soybeans with supercritical carbon dioxide and ethanol. J. Supercritical Fluids. v. 14, p. 87-93, 1999.

[14] S.L. Melton, R.E. Moyers, E C.G. Playford, Lipids Extracted from Soy Products by Different Procedures. J. Am. Oil Chem. Soc., v. 56, p. 489-493, 1979.

[15] K. Nielsen, The Composition of the Difficultly Extractable Soybean Phosphatides. J. Am. Oil Chem. Soc., v. 37. p. 217219, 1960.

[16] L.P. Hayes, R.P Simms. Defatted soybean fractionation by solvent extraction. U.S. 3.737.901. 22 Mai 1973.

[17] A.A.S Brum, L.F Arruda, M.A.B.R Darce. Métodos de extração e qualidade da fração lipídica de matérias-primas de origem vegetal e animal Quim. Nova, Vol. 32, No. 4, 849-854, 2009

[18] AMERICAN OIL CHEMISTS' SOCIETY - AOCS, Official methods and recommended pratices of the AOCS, 2004. AOCS PRESS. ISBN 1-893997-48-0. United States.

[19] E.W. Lusas, E K.C. Rhee, Soy protein processing and utilization. In: ERICKSON, D.R. Pratical handbook of soybean processing and utilization. St. Louis: AOCS PRESS and the United Soybean Board, 1995. P. 117-160.

[20] A. P. Gandhi, K. C. Joshi, J. Krishna, D. C. Paribar, P. R Srivastav, J. Kawalkar, S. K. Jain, R. N. Tripathi, Studies on alternative solvents for the extraction of oil-I soybean Int. J. Food Sci. Technol. 2003, 38, 369

[21] F. Chemat, Z. H. Muhammed K. Khan F. et al. Applications of ultrasound in food technology: processing, preservation and extraction. Ultrasonics Sonochemistry, v.18, n.4, p.813-835, 2011.

[22] Y. Wu, T. Wang Soybean lecithin fractionation and functionality. Journal of the American Oil Chemists' Society v. 80, n.4, p.319-326, 2003

[23] R. Kaparthi, E K.S. Chari, Solubilities of vegetable oils in aqueous ethanol and ethanol-hexane mixtures. J. Am. Oil Chem. Soc., v. 36, n. 2, p. 77-80, 1959. 